\title{
The effects of probiotics on body weight and biomarkers of animal
}

\begin{abstract}
Obesity is associated with the alteration of the gut microbiota. In order to determine the effects of probiotics on body weight management and its related biomarkers we performed a systematic review using clinical trial, interventional and experimental studies. We performed a broad search with no date restriction. Primary outcomes were included the parameters related to body weight management. Secondary outcomes were inflammatory markers, lipid profile, blood glucose and insulin level. A total of 12 animal studies were identified. Among these, six studies reported the significant changes in body weight and all the studies had documented significant improvements in at least one body weight related parameter. However, inflammatory markers and lipid profile were significantly improved in the animal model; changes in body weight and energy intake that could be due to probiotics supplementation were controversial. Different strains of gut microbiota have different effects on weight changes. Further studies are needed to identify the role of gut microbiota on weight regulation of human.
\end{abstract}

Keyword: Gut microbiota; Probiotic; Weight regulation 